\title{
ASYMPTOTIC BEHAVIOUR OF RANDOM MARKOV CHAINS WITH TRIDIAGONAL GENERATORS
}

\author{
PETER E. KLOEDEN ${ }^{\bowtie}$ and VICTOR S. KOZYAKIN
}

(Received 25 January 2012)

\begin{abstract}
Continuous-time discrete-state random Markov chains generated by a random linear differential equation with a random tridiagonal matrix are shown to have a random attractor consisting of singleton subsets, essentially a random path, in the simplex of probability vectors. The proof uses comparison theorems for Carathéodory random differential equations and the fact that the linear cocycle generated by the Markov chain is a uniformly contractive mapping of the positive cone into itself with respect to the Hilbert projective metric. It does not involve probabilistic properties of the sample path and is thus equally valid in the nonautonomous deterministic context of Markov chains with, say, periodically varying transition probabilities, in which case the attractor is a periodic path.
\end{abstract}

2010 Mathematics subject classification: primary 34F05; secondary 37H10, 60H25, 60J10, 15B48, 15B51, 15B52.

Keywords and phrases: random differential equations, random Markov chains, positive cones, uniformly contracting cocycles, random attractors.

\section{Introduction}

The simplex $\Sigma_{N}$ in $\mathbb{R}^{N}$ defined by

$$
\Sigma_{N}=\left\{\boldsymbol{p}=\left(p_{1}, \ldots, p_{N}\right)^{T}: \sum_{j=1}^{N} p_{j}=1, p_{1}, \ldots, p_{N} \in[0,1]\right\}
$$

is invariant under the dynamics of the system of ordinary differential equations

$$
\frac{d \boldsymbol{p}}{d t}=Q \boldsymbol{p}
$$

P. E. Kloeden is partially supported by DFG grant KL 1203/7-1, the Spanish Ministerio de Ciencia e Innovación project MTM2011-22411, the Consejería de Innovación, Ciencia y Empresa (Junta de Andalucía) under the Ayuda 2009/FQM314 and the Proyecto de Excelencia P07-FQM-02468. V. S. Kozyakin is partially supported by the Russian Foundation for Basic Research, project no. 1001-93112.

(c) 2012 Australian Mathematical Publishing Association Inc. 0004-9727/2012 \$16.00 
in $\mathbb{R}^{N}$, where the $N \times N$ matrix $Q$ satisfies

$$
\mathbf{1}_{N}^{T} Q \equiv \mathbf{0}
$$

Here $\mathbf{1}_{N}$ is the column vector in $\mathbb{R}^{N}$ with all components equal to 1 .

System (1.1) restricted to $\Sigma_{N}$ generates a continuous-time finite-state Markov chain with a generator $Q$. Such Markov chains are common in the biological sciences with generators given by an $N \times N$ tridiagonal matrix

$$
Q=\left[\begin{array}{ccccccr}
-q_{1} & q_{2} & & & & & \\
q_{1} & -\left(q_{2}+q_{3}\right) & q_{4} & & & & \\
& \ddots & \ddots & \ddots & \ddots & \ddots & \\
& & & & q_{2 N-5} & -\left(q_{2 N-4}+q_{2 N-3}\right) & q_{2 N-2} \\
& & & & & q_{2 N-3} & -q_{2 N-2}
\end{array}\right]
$$

with positive off-diagonal components $\left\{q_{i}\right\}$; see $[1,4,15]$. The components of $Q$ may depend on time or be random, or both, that is, depend on a stochastic process, in which case $Q$ will be written $Q\left(\theta_{t} \omega\right)$ (the undefined terms will be explained later).

The asymptotic behaviour of the discrete-time version of such Markov chains was investigated by the authors in [5]. Their transition matrices are of the form $I_{N}+Q\left(t_{n}\right) \Delta_{n}$, where $I_{N}$ is the $N \times N$ identity matrix, $Q\left(t_{n}\right)$ is the value of the generator matrix $Q$ at time $t_{n}$, that is, $Q\left(t_{n}\right)=Q\left(\theta_{t_{n}} \omega\right)$, and $\Delta_{n}$ is a positive parameter. This is just the Euler scheme for the differential equation (1.1) on $\Sigma_{N}$ with the time step $\Delta_{n}$. It was shown in [5] that these discrete-time Markov chains generate a linear random dynamical system which has a random attractor consisting of singleton components sets, essentially a random process of probability vectors in $\Sigma_{N}$.

In this paper the corresponding result will be established for continuous-time finitestate Markov chains generated by (1.1) in $\Sigma_{N}$, where $Q$ is an $N \times N$ tridiagonal matrix of the form (1.2) with components driven by a stochastic process. This will be proved directly. An indirect proof using the convergence of the random attractors of the Euler scheme requires them to have a uniform rate of attraction. This is the inverse problem of what is usually discussed in numerical dynamics, where it is shown that the numerical scheme preserves the properties of continuous-time systems generated by the differential equation; see [6, 12].

The paper is structured as follows. Basic properties of differential equations satisfying the Carathéodory property are recalled in Section 2. In particular, in Section 2.1, properties of deterministic linear differential equations of the type (1.1) are investigated, while in Section 2.2 some notions on related random differential equations (RDEs) of the type (1.1) are recalled. Finally, in Section 2.3 it is shown that that the related RDE preserves the positivity of initial values and in Section 3 the main result of the paper is presented, a theorem on the existence of a random attractor consisting of singleton component subsets in the system under consideration. 


\section{Carathéodory differential equations}

The linear ordinary differential equation (1.1) with the matrix $Q$ depending on a stochastic process is a differential equation of the Carathéodory type. Some basic properties of such equations are recalled here along with some comparison theorems, which will be used to establish the positivity of solutions of the resulting random differential equation.

An ordinary differential equation on $\mathbb{R}^{N}$,

$$
\frac{d \boldsymbol{x}}{d t}=f(t, \boldsymbol{x})
$$

is called a Carathéodory differential equation if the vector-field $f(t, \boldsymbol{x})$ satisfies the Carathéodory conditions:

(C1) for every fixed $t$, the function $\boldsymbol{x} \mapsto f(t, \boldsymbol{x})$ is continuous;

(C2) for every fixed $\boldsymbol{x}$, the function $t \mapsto f(t, \boldsymbol{x})$ is measurable in $t$ and there exists a function $m_{x}(t)$, which is Lebesgue integrable on every bounded subinterval, such that $\|f(t, x)\| \leq m_{x}(t)$.

A solution of (2.1) is a vector-valued function $\boldsymbol{x}(t)$ which is absolutely continuous on some interval and satisfies (2.1) almost everywhere on this interval. The existence and uniqueness of solutions of (2.1) on the whole real axis $\mathbb{R}$ follow from the Carathéodory conditions if, in addition, the function $f(t, \boldsymbol{x})$ is defined for all $(t, \boldsymbol{x}) \in$ $\mathbb{R}^{1} \times \mathbb{R}^{N}$ and satisfies a local Lipschitz condition in $\boldsymbol{x}$ in a neighbourhood of every initial point $\left(t_{0}, \boldsymbol{x}_{0}\right)$.

A solution $\boldsymbol{x}(t)=\left(x_{1}(t), x_{2}(t), \ldots, x_{N}(t)\right)^{\top}$ of (2.1) is called positive (strongly positive) if

$$
x_{i}(t) \geq 0(>0) \quad \text { for all } t \text { and } i=1,2, \ldots, N .
$$

The next condition guarantees the positivity of solutions of (2.1); see, for example, [7, 11, 13, 14]. A function $f=\left(f_{1}, f_{2}, \ldots, f_{N}\right)^{\top}: \mathbb{R}^{1} \times \mathbb{R}^{N} \rightarrow \mathbb{R}^{N}$ is called quasipositive, or off-diagonal positive, if, for each $i=1,2, \ldots, N$,

$$
f_{i}\left(t, x_{1}, \ldots, x_{i-1}, 0, x_{i+1}, \ldots, x_{N}\right) \geq 0,
$$

whenever $x_{j} \geq 0$ for $j \neq i$. A function $f=\left(f_{1}, f_{2}, \ldots, f_{N}\right)^{\top}$ is called strongly quasipositive, or strongly off-diagonal positive, if, for each $i=1,2, \ldots, N$,

$$
f_{i}\left(t, x_{1}, \ldots, x_{i-1}, 0, x_{i+1}, \ldots, x_{N}\right)>0,
$$

whenever $x_{j} \geq 0$ for all $j$ and $\sum_{j} x_{j}>0$.

The proof of the following theorem for the case where $f(t, \boldsymbol{x})$ is continuous in $t$ and $\boldsymbol{x}$ can be found, for example, in [7, Lemma 4.1]. It was noted in [13] that similar statements are also valid when $f(t, \boldsymbol{x})$ satisfies the Carathéodory conditions.

THEOREM 2.1. If the vector-field $f$ of (2.1) is quasipositive, then the solution $\boldsymbol{x}(t)$ of (2.1) satisfying the initial condition $\boldsymbol{x}(0)=\boldsymbol{x}_{0}=\left(x_{0,1}, x_{0,2}, \ldots, x_{0, N}\right)$ is positive for $t \geq 0$ whenever $x_{0, i} \geq 0$ for $i=1,2, \ldots, N$. 
If the vector-field $f$ of (2.1) is strongly quasipositive, then the solution $\boldsymbol{x}(t)$ of (2.1) satisfying the initial condition $\boldsymbol{x}(0)=\boldsymbol{x}_{0}=\left(x_{0,1}, x_{0,2}, \ldots, x_{0, N}\right)$ is strongly positive for $t \geq 0$ whenever $x_{0, i}>0$ for $i=1,2, \ldots, N$.

2.1. Deterministic linear differential equations. Unfortunately the requirement that a vector-field function be quasipositive is rather restrictive and does not apply to a deterministic linear differential equation

$$
\frac{d \boldsymbol{p}}{d t}=Q \boldsymbol{p}, \quad \boldsymbol{p} \in \mathbb{R}^{N},
$$

with a tridiagonal matrix $Q$ given by (1.2).

There is, however, another useful notion that can be used in combination with it. An $N \times N$ matrix $Q$ with nonnegative off-diagonal elements is said to have a path of nonsingularity [7] if there is a set of indices $i_{1}, i_{2}, \ldots, i_{n}$ with $i_{n}=i_{1}$ such that all the elements $q_{i_{j}, i_{j+1}}$ are strictly positive and this set of indices contains all the numbers 1 , $2, \ldots, N$. In particular, the matrix $Q$ defined by (1.2) has the path of nonsingularity

$$
\left\{i_{1}, i_{2}, \ldots, i_{2 N-1}\right\}=\{1,2, \ldots, N-1, N, N-1, \ldots, 2,1\} .
$$

Recall that a set $K$ in a Banach space is called a cone if it is convex, closed with $t K \subseteq K$ for any real $t \geq 0$ and $K \cap-K=\{0\}$; see, for example [8]. Fix a norm $\|\cdot\|$ in $\mathbb{R}^{N}$, and denote by $\mathbb{K}_{+}^{N}$ the cone of elements $x=\left(x_{1}, x_{2}, \ldots, x_{N}\right)^{\top} \in \mathbb{R}^{N}$ with nonnegative components, and by $\stackrel{\circ}{\mathbb{K}}_{+}^{N}$ the interior of $\mathbb{K}_{+}^{N}$, which is clearly nonempty. In addition, denote by $\mathbb{S}^{N}$ the unit ball in the norm $\|\cdot\|$.

THEOREM 2.2. Suppose that the matrix $Q$ in (2.2) with nonnegative off-diagonal entries has a path of nonsingularity. Then, for any nonzero initial condition $\boldsymbol{p}(0)=\boldsymbol{p}_{0} \in \mathbb{K}_{+}^{N}$, the solution $\boldsymbol{p}\left(t, \boldsymbol{p}_{0}\right)$ is strongly positive for all $t>0$.

Moreover, for every bounded interval $\left[T_{1}, T_{2}\right] \subset(0, \infty)$, there is a number $\alpha\left(T_{1}, T_{2}\right)>0$ such that

$$
\boldsymbol{p}\left(t ; \boldsymbol{p}_{0}\right)+\alpha\left(T_{1}, T_{2}\right)\left\|\boldsymbol{p}_{0}\right\| \mathbb{S}^{N} \subseteq \mathbb{K}_{+}^{N}
$$

for all $\boldsymbol{p}_{0} \in \mathbb{K}_{+}^{N} \backslash\{0\}$ and $t \in\left[T_{1}, T_{2}\right]$.

Proof. The first part of Theorem 2.2 follows from [7, Theorem 4.7], while the second part is clear modulo the first part.

Remark 2.3. The inclusion (2.3) means that the solution operator $\boldsymbol{p}_{0} \mapsto \boldsymbol{p}\left(t, \boldsymbol{p}_{0}\right)$ maps the set $\mathbb{K}_{+}^{N} \backslash\{0\}$ into its interior $\stackrel{\circ}{\mathbb{K}}_{+}^{N}$ for every $t>0$.

Remark 2.4. If the matrix $Q$ in (2.2) satisfies the conditions of Theorem 2.2 as well as $\mathbf{1}_{N}^{T} Q=\mathbf{0}$, then $\boldsymbol{p}\left(t ; \Sigma_{N}\right) \subseteq \Sigma_{N}$ for all $t \geq 0$. Moreover, the simplex $\Sigma_{N}$ is mapped by $\boldsymbol{p}(t ; \cdot)$ into its interior $\stackrel{\circ}{\Sigma}_{N}$ for every $t>0$. 
2.2. Random linear differential equations. Let $(\Omega, \mathcal{F}, \mathbb{P})$ be a probability space. In the theory of random dynamical systems [2, 3] the noise process that drives the dynamics is given in terms of an abstract metric dynamical system.

Definition 2.5. A metric dynamical system $\theta \equiv\left\{\theta_{t}\right\}_{t \in \mathbb{R}}$ on a probability space $(\Omega, \mathcal{F}, \mathbb{P})$ is a family of transformations $\theta_{t}: \Omega \rightarrow \Omega, t \in \mathbb{R}$, such that:

(1) it is a one-parameter group, that is,

$$
\theta_{0}=\mathrm{id}_{\Omega}, \quad \theta_{t} \circ \theta_{s}=\theta_{t+s} \quad \text { for all } t, s \in \mathbb{R}
$$

(2) $\quad(t, \omega) \mapsto \theta_{t} \omega$ is jointly measurable;

(3) $\theta_{t} \mathbb{P}=\mathbb{P}$ for all $t \in \mathbb{R}$, that is, $\mathbb{P}\left(\theta_{t} B\right)=\mathbb{P}(B)$ for all $B \in \mathcal{F}$ and $t \in \mathbb{R}$.

Consider the random linear differential equation $[2,3]$

$$
\frac{d \boldsymbol{p}}{d t}=Q\left(\theta_{t} \omega\right) \boldsymbol{p}, \quad \boldsymbol{p}(0)=\boldsymbol{p}_{0} \in \mathbb{R}^{N},
$$

driven by the metric dynamical system $\theta$ on a probability space $(\Omega, \mathcal{F}, \mathbb{P})$, where the coefficients $q_{j}: \Omega \rightarrow \mathbb{R}$ in the $N \times N$ matrix $Q$ are locally bounded $\mathcal{F}$-measurable functions.

More precisely, assume that there exists a random variable $C$ with $C(\omega)>0$ such that

$$
\int_{\alpha}^{\alpha+1} C\left(\theta_{t} \omega\right) d t<\infty \quad \text { for all } \alpha \in \mathbb{R}
$$

and

$$
\left|q_{i}(\omega)\right| \leq C(\omega) \text { for } i=1,2, \ldots, 2 N-2
$$

for all $\omega \in \Omega$. It is to be emphasised that assumptions (2.5) and (2.6) are stated here for all $\omega \in \Omega$. As explained in [3, p. 56] this does not restrict generality.

Remark 2.6. Conditions (2.5) and (2.6) are satisfied if

$$
\left|q_{i}(\omega)\right| \leq C_{*} \quad \text { for all } i \text { and } \omega \in \Omega
$$

for some constant $C_{*}>0$.

The random differential equation (2.4) is thus a Carathéodory ordinary differential equation for each sample path. For each $\omega \in \Omega$ the vector-field mapping $f(t, \boldsymbol{p})=$ $Q\left(\theta_{t} \omega\right) \boldsymbol{p}$ satisfies the Carathéodory conditions (2.5) and (2.6) and is linear and thus globally Lipschitz in $\boldsymbol{p}$. It follows that the initial value problem (2.4) has a unique global solution $\boldsymbol{p}(t, \omega) \equiv \boldsymbol{p}\left(t, \omega ; \boldsymbol{p}_{0}\right)$ for any initial value $\boldsymbol{p}_{0} \in \mathbb{R}^{N}$, where this solution exists for all $t \geq 0$. (See [3] for more details, explicitly in terms of random differential equations.) Moreover, this solution depends continuously on $\boldsymbol{p}_{0}$ for each $\omega \in \Omega$.

It will now be shown that (2.4) preserves the positivity of initial values. 
2.3. Positivity of solutions. The results from the previous sections will now be applied to the RDE in $\mathbb{R}^{N}$ given by

$$
\frac{d \boldsymbol{p}}{d t}=Q\left(\theta_{t} \omega\right) \boldsymbol{p}, \quad \boldsymbol{p}(0)=\boldsymbol{p}_{0} \in \mathbb{R}^{N},
$$

driven by a metric dynamical system $\theta$, where the matrix $Q(\omega)$ is of the tridiagonal form (1.2) and satisfies

$$
\mathbf{1}_{N}^{T} Q(\omega) \equiv \mathbf{0} \quad \text { for all } \omega \in \Omega .
$$

Suppose further that the coefficients in the matrix $Q(\omega)$ are locally bounded $\mathcal{F}$ measurable functions satisfying uniform bounds

$$
0<c_{*} \leq q_{i}(\omega) \leq C_{*}<\infty, \quad i=1,2, \ldots, 2 N-2, \omega \in \Omega .
$$

Then, by Remark 2.6, $Q(\omega)$ satisfies the conditions (2.5) and (2.6).

Define (a deterministic) tridiagonal matrix

$$
\bar{Q}=\left[\begin{array}{ccccccc}
\tilde{q}_{1} & \bar{q}_{2} & & & & & \\
\bar{q}_{1} & \tilde{q}_{2} & \bar{q}_{4} & & & & \\
& \ddots & \ddots & \ddots & \ddots & \ddots & \\
& & & & \bar{q}_{2 N-5} & \tilde{q}_{N-1} & \bar{q}_{2 N-2} \\
& & & & & \bar{q}_{2 N-3} & \tilde{q}_{N}
\end{array}\right]
$$

with the off-diagonal elements $\bar{q}_{i}$ given by

$$
\bar{q}_{i}=\inf _{\omega \in \Omega} q_{i}(\omega), \quad i=1,2, \ldots, 2 N-2,
$$

and the diagonal elements $\tilde{q}_{i}$ satisfying

$$
\begin{gathered}
\tilde{q}_{1}=\inf _{\omega \in \Omega}\left\{-q_{1}(\omega)\right\}, \\
\tilde{q}_{i}=\inf _{\omega \in \Omega}\left\{-\left(q_{2 i-2}(\omega)+q_{2 i-1}(\omega)\right)\right\}, \quad i=2, \ldots, 2 N-1, \\
\tilde{q}_{N}=\inf _{\omega \in \Omega}\left\{-q_{2 N-2}(\omega)\right\} .
\end{gathered}
$$

Then

$$
Q(\omega) \geq \bar{Q} \text { for all } \omega \in \Omega,
$$

where the inequality between the matrices is interpreted componentwise. The offdiagonal elements $\tilde{q}_{i}$ of the matrix $\bar{Q}$ are strictly positive since

$$
0<c_{*} \leq \bar{q}_{i}, \quad i=1,2, \ldots, 2 N-2 .
$$

Consider the differential equation

$$
\frac{d \boldsymbol{q}}{d t}=\bar{Q} \boldsymbol{q}
$$

and denote the solution of this equation with the initial condition $\boldsymbol{q}(0)=\boldsymbol{q}_{0} \in \mathbb{R}^{N}$ by $\boldsymbol{q}\left(t ; \boldsymbol{q}_{0}\right)$. 
REMARK 2.7. It follows from (2.10) that $\bar{Q}$ is a matrix with nonnegative off-diagonal elements that has a path of nonsingularity. Then by Theorem 2.2 and Remark 2.3 the solution operator $\boldsymbol{q}(t ; \cdot)$ maps the set $\mathbb{K}_{+}^{N} \backslash\{0\}$ into its interior $\stackrel{\circ}{\mathbb{K}}_{+}^{N}$ for each $t>0$. Moreover, relations of the type (2.3) hold for it with appropriate parameters.

Remark 2.8. The relation $\mathbf{1}_{N}^{T} \bar{Q} \equiv \mathbf{0}$ is not valid in general. Then the simplex $\Sigma_{N}$ will not be invariant under $\boldsymbol{q}(t ; \cdot)$, but this will be of no importance in what follows.

Theorem 2.9. Let $\theta$ be a metric dynamical system and let $Q(\omega)$ be a matrix of the tridiagonal form (1.2), which satisfies (2.8) and (2.9). Then the solution $\boldsymbol{p}\left(t ; \omega, \boldsymbol{p}_{0}\right)$ of the random linear differential equation (2.7) satisfies

$$
\boldsymbol{p}\left(t ; \omega, \boldsymbol{p}_{0}\right) \geq \boldsymbol{q}\left(t ; \boldsymbol{p}_{0}\right) \quad \text { for all } \omega \in \Omega, t \geq 0, \boldsymbol{p}_{0} \in \mathbb{K}_{+}^{N},
$$

where the inequality is meant componentwise.

Proof. Fix an $\omega \in \Omega$ and fix the initial conditions $\boldsymbol{p}(0)=\boldsymbol{q}(0)=\boldsymbol{p}_{0} \in \mathbb{K}_{+}^{N}$ for the differential equations (2.7) and (2.11), respectively. Then the function

$$
\boldsymbol{x}(t):=\boldsymbol{p}\left(t ; \omega, \boldsymbol{p}_{0}\right)-\boldsymbol{q}\left(t ; \boldsymbol{p}_{0}\right)
$$

satisfies the differential equation

$$
\frac{d \boldsymbol{x}}{d t}=Q\left(\theta_{t} \omega\right) \boldsymbol{p}-\bar{Q} \boldsymbol{q}=Q\left(\theta_{t} \omega\right) \boldsymbol{x}+\left(Q\left(\theta_{t} \omega\right)-\bar{Q}\right) \boldsymbol{q}
$$

with the initial condition $\boldsymbol{x}(0)=0$. Denoting

$$
\boldsymbol{w}(t):=\left(Q\left(\theta_{t} \omega\right)-\bar{Q}\right) \boldsymbol{q}\left(t ; \boldsymbol{p}_{0}\right),
$$

this differential equation can be written as

$$
\frac{d \boldsymbol{x}}{d t}=Q\left(\theta_{t} \omega\right) \boldsymbol{x}+\boldsymbol{w}(t), \quad \boldsymbol{x}(0)=0 .
$$

The matrix $Q\left(\theta_{t} \omega\right)-\bar{Q}$ has, by definition, nonnegative components for all $t$ and $\omega$. By Remark 2.7 the function $\boldsymbol{q}\left(t ; \boldsymbol{p}_{0}\right)$ with $\boldsymbol{p}_{0} \in \mathbb{K}_{+}^{N}$ has nonnegative components for all $t \geq 0$. Hence the function $\boldsymbol{w}(t)$ has nonnegative components. Moreover, the matrix $Q(\omega)$ as a matrix with nonnegative off-diagonal components has a path of nonsingularity, so it follows that (2.13) satisfies all the conditions of Theorem 2.1. Hence, the solution function $\boldsymbol{x}(t)$ is positive, that is, its components are nonnegative.

Theorem 2.9 has some important consequences.

Corollary 2.10. The following statements are valid:

(i) For any $\omega \in \Omega$ and $t>0$, the solution operator $\boldsymbol{p}(t ; \omega, \cdot)$ maps the set $\mathbb{K}_{+}^{N} \backslash\{0\}$ into its interior $\stackrel{\circ}{\mathbb{K}_{+}^{N}}$. 
(ii) For any $\omega \in \Omega$ and $t>0$, the solution operator $\boldsymbol{p}(t ; \omega, \cdot)$ maps the simplex $\Sigma_{N}$ into its interior $\stackrel{\circ}{\Sigma}_{N}$.

(iii) For any bounded interval $\left[T_{1}, T_{2}\right] \subset(0, \infty)$ there is a number $\beta\left(T_{1}, T_{2}\right)>0$ such that

$$
\boldsymbol{p}\left(t ; \omega, \boldsymbol{p}_{0}\right)+\beta\left(T_{1}, T_{2}\right)\left\|\boldsymbol{p}_{0}\right\|\left(\mathbb{S}^{N} \cap \Sigma_{N}\right) \subseteq \Sigma_{N},
$$

for all $\omega \in \Omega, p_{0} \in \mathbb{K}_{+}^{N} \backslash\{0\}, t \in\left[T_{1}, T_{2}\right]$.

To prove Corollary 2.10 it suffices to note that (i) follows immediately from (2.12) and Remark 2.3. This, due to (2.8), implies (ii). Then (i) and (ii), together with the inclusion (2.3) valid for the solution operator $\boldsymbol{q}(t ; \cdot)$, imply (iii).

\section{Random dynamical systems}

Let $(\Omega, \mathcal{F}, \mathbb{P})$ be a probability space. A random dynamical system [2, 3] has a skew product structure consisting of a metric dynamical system (2.5) that models the driving noise process and a cocycle mapping.

Let $M$ be a complete metric space equipped with the metric $\rho$ and let $\theta$ be a metric dynamical system.

Definition 3.1. A map $\varphi: \mathbb{R}^{+} \times \Omega \times M \rightarrow M$ is called a cocycle on $M$ with respect to the driving system $\theta$ if it satisfies the initial condition

$$
\varphi(0, \omega, \cdot)=\mathrm{id}_{X} \quad \text { for all } \omega \in \Omega,
$$

and the cocycle property

$$
\varphi(t+s, \omega, x)=\varphi\left(s, \theta_{t} \omega, \varphi(t, \omega, x)\right) \quad \text { for all } x \in M, \omega \in \Omega, t, s \in \mathbb{R}^{+} .
$$

The RDE (2.4) generates a random dynamical system $(\theta, \varphi)$ on $\mathbb{R}^{N}$ with the cocycle mapping $\varphi$ defined by

$$
\varphi\left(t, \omega, \boldsymbol{p}_{0}\right)=\boldsymbol{p}\left(t, \omega ; \boldsymbol{p}_{0}\right) \quad \text { for all } t \geq 0, \omega \in \Omega, \boldsymbol{p}_{0} \in \mathbb{R}^{N} .
$$

In fact, the mapping $(t, \boldsymbol{p}) \mapsto \varphi(t, \omega, \boldsymbol{p})$ is continuous for each $\omega \in \Omega$, while the mapping $\boldsymbol{p} \mapsto \varphi(t, \omega, \boldsymbol{p})$ is linear.

3.1. Random attractors. The asymptotic behaviour of a random dynamical system is described by random attractors.

Definition 3.2. A random attractor $\mathscr{A}=\{A(\omega), \omega \in \Omega\}$ of a random dynamical system $(\theta, \varphi)$ is a family of nonempty compact subsets of $M$ such that:

(1) the set-valued mapping $\omega \mapsto A(\omega)$ is $\mathcal{F}$-measurable;

(2) $\mathscr{A}$ is $\varphi$-invariant, that is,

$$
\varphi(t, \omega, A(\omega))=A\left(\theta_{t} \omega\right)
$$

for every $\omega \in \Omega$ and $t \in \mathbb{R}^{+}$; 
(3) $\mathscr{A}$ pullback attracts $\mathcal{F}$-measurable families $\mathscr{D}=\{D(\omega), \omega \in \Omega\}$ of nonempty compact subsets of $M$, that is,

$$
\operatorname{dist}\left(\varphi\left(t, \theta_{-t} \omega, D\left(\theta_{-t} \omega\right)\right), A(\omega)\right) \rightarrow 0 \quad \text { as } t \rightarrow 0
$$

for every $\omega \in \Omega$.

It follows from Theorem 2.9 and Corollary 2.10 that the solution operator $\boldsymbol{p}(t ; \omega, \cdot)$ of the RDE (2.4) maps the simplex $\Sigma_{N}$ into its interior $\stackrel{\circ}{\Sigma}_{N}$ uniformly for $t$ in bounded intervals from $(0, \infty)$.

The Hilbert projective metric $\rho_{H}$ on $\mathbb{K}_{+}^{N}$ will be used. It can be defined as

$$
\rho_{H}(x, y)=\left|\log \left(\frac{\max _{i} y_{i} / x_{i}}{\max _{i} x_{i} / y_{i}}\right)\right|
$$

for vectors $x=\left(x_{1}, x_{2}, \ldots, x_{N}\right)^{\top}$ and $y=\left(y_{1}, y_{2}, \ldots, y_{N}\right)^{\top}$ in $\mathbb{K}_{+}^{N}$. It is in fact only a semi-metric on $\mathbb{K}_{+}^{N}$, but becomes a metric on a projective space. It is important here that the interior $\stackrel{\circ}{\Sigma}_{N}$ of the probability simplex $\Sigma_{N}$ is the complete metric space with the Hilbert projective metric. (See, for example, [8-10] and the references therein, on the properties of the Hilbert projective metric.) In the Russian literature the Hilbert projective metric is also called the Birkhoff metric.

The main result of this paper, Theorem 3.4 below, requires the following definitions.

Definition 3.3. A cocycle mapping $\varphi: \mathbb{R}^{+} \times \Omega \times M \rightarrow M$, where $(M, \rho)$ is a metric space, is called uniformly dissipative if there exist a number $T_{d} \in \mathbb{R}^{+}$and a closed bounded set $M_{0} \subset M$ such that

$$
\varphi\left(T_{d}, \omega, M\right) \subseteq M_{0}, \quad \text { for all } \omega \in \Omega .
$$

It is called uniformly contractive if there exist a number $T_{c} \in \mathbb{R}^{+}$and a number $\lambda \in[0,1)$ such that

$$
\rho\left(\varphi\left(T_{c}, \omega, x\right), \varphi\left(T_{c}, \omega, y\right)\right) \leq \lambda \rho(x, y), \quad \text { for all } \omega \in \Omega, x, y \in M .
$$

THEOREM 3.4. The restriction of $\boldsymbol{p}(t ; \omega, \cdot)$ to the set $\Sigma_{N}$ is a uniformly dissipative and uniformly contractive cocycle (with respect to the Hilbert projective metric), which has a random attractor $\mathscr{A}=\{A(\omega), \omega \in \Omega\}$ such that set $A(\omega)=\{a(\omega)\}$ consists of a single point for each $\omega \in \Omega$. Moreover, the random attractor is asymptotically stable, that is,

$$
\left\|\boldsymbol{p}\left(t, \omega, \boldsymbol{p}_{0}\right)-a\left(\theta_{t} \omega\right)\right\| \rightarrow 0 \quad \text { as } t \rightarrow \infty
$$

for all $\boldsymbol{p}_{0} \in \stackrel{\circ}{\Sigma}_{N}$ and $\omega \in \Omega$.

PRoof. The proof follows by an application of the discrete-time case in [5] to, for example, the time-one mapping of the RDE, that is, the cocycle mapping at integer time values. The uniform dissipativity of the cocycle ensures the existence of a random attractor, while the uniform contractivity implies that the component subsets are singleton sets. 


\section{References}

[1] L. J. S. Allen, An Introduction to Stochastic Processes with Applications to Biology, 2nd edn (CRC Press, Boca Raton, FL, 2011).

[2] L. Arnold, Random Dynamical Systems, Springer Monographs in Mathematics (Springer, Berlin, 1998).

[3] I. Chueshov, Monotone Random Systems Theory and Applications, Lecture Notes in Mathematics, 1779 (Springer, Berlin, 2002).

[4] A. E. Hutzenthaler, Mathematical Models for Cell-cell Communication on Different Time Scales, $\mathrm{PhD}$ Thesis, Zentrum Mathematik, Technische Universität München, 2009.

[5] P. E. Kloeden and V. S. Kozyakin, 'Asymptotic behaviour of random tridiagonal Markov chains in biological applications', Discrete Contin. Dyn. Syst. Ser. B. to appear.

[6] P. E. Kloeden and J. Lorenz, 'Stable attracting sets in dynamical systems and in their one-step discretizations', SIAM J. Numer. Anal. 23(5) (1986), 986-995.

[7] M. A. Krasnosel'skiū, The Operator of Translation along the Trajectories of Differential Equations, Translations of Mathematical Monographs, 19 (American Mathematical Society, Providence, RI, 1968), translated from the Russian by Scripta Technica.

[8] M. A. Krasnosel'skij, Je. A. Lifshits and A. V. Sobolev, Positive Linear Systems: The Method of Positive Operators, Sigma Series in Applied Mathematics, 5 (Heldermann, Berlin, 1989), translated from the Russian by Jürgen Appell.

[9] R. D. Nussbaum, 'Some nonlinear weak ergodic theorems', SIAM J. Math. Anal. 21(2) (1990), 436-460.

[10] R. D. Nussbaum, 'Finsler structures for the part metric and Hilbert's projective metric and applications to ordinary differential equations', Differ. Integral Equ. 7(5-6) (1994), 1649-1707.

[11] H. L. Smith, Monotone Dynamical Systems: An Introduction to the Theory of Competitive and Cooperative Systems, Mathematical Surveys and Monographs, 41 (American Mathematical Society, Providence, RI, 1995).

[12] A. M. Stuart and A. R. Humphries, Dynamical Systems and Numerical Analysis, Cambridge Monographs on Applied and Computational Mathematics, 2 (Cambridge University Press, Cambridge, 1996).

[13] J. Szarski, Differential Inequalities, Monografie Matematyczne, Tom 43 (Państwowe Wydawnictwo Naukowe, Warsaw, 1965).

[14] W. Walter, 'Differential inequalities', in: Inequalities (Birmingham, 1987), Lecture Notes in Pure and Applied Mathematics, 129 (Dekker, New York, 1991), pp. 249-283.

[15] D. Wodarz and N. Komarova, Computational Biology of Cancer: Lecture Notes and Mathematical Modeling (World Scientific, Singapore, 2005).

PETER E. KLOEDEN, Institut für Mathematik, Goethe Universität, D-60054 Frankfurt am Main, Germany

e-mail: kloeden@math.uni-frankfurt.de

VICTOR S. KOZYAKIN, Institute for Information Transmission Problems, Russian Academy of Sciences, Bolshoj Karetny lane, 19, 101447 Moscow, Russia e-mail: kozyakin@iitp.ru 